\title{
The Influence of Ankle Braces on Functional Performance Tests and Ankle Joint Range of Motion
}

\author{
Brittany Mann, Allison H. Gruber, Shane P. Murphy, and Carrie L. Docherty \\ Indiana University
}

To be published in Journal of Sport Rehabilitation

\begin{abstract}
Context: The lateral ankle sprain is one of the most common lower extremity injuries in sports. Previous research has found that some prophylactic ankle supports reduce the risk of recurrent ankle sprains and provide extra support to the joint. However, there is a continued concern that these supports may negatively influence performance. Objective: To determine if wearing an ankle brace influences athlete performance and ankle kinematics during functional performance tests (FPTs). Design: Repeated measures. Setting: University Gymnasium. Other Participants: Male and female recreational or competitive athletes $(\mathrm{n}=20)$. Intervention: Participants performed three trials of a standing long jump, vertical jump, 40 yard sprint, and T-drill under each of the following three conditions: wearing traditional lace-up brace (brace 1), modified lace-up brace (brace 2), and no-brace. Main Outcome Measures: A 2D motion capture camera was used to measure ankle range of motion (ROM) in the sagittal plane during the vertical and standing long jumps and in the frontal plane during the cutting phase of the T-drill. Performance of each test and ankle ROM were compared between each of the braced conditions. Results: Ankle braces did not influence performance in speed or agility FPTs ( $>0.05)$. Ankle braces negatively affected performance of the standing long jump $(\mathrm{p}=0.01)$ and vertical jump $(\mathrm{p}=0.01)$. There was no significant difference between brace or no-brace conditions in ankle inversion ROM during the T-drill ( $>00.05)$. Both brace conditions restricted ROM in the sagittal plane during the vertical and standing long jumps $(\mathrm{p}<0.05)$. Conclusions: Braced conditions restricted
\end{abstract}


sagittal plane ROM during the vertical jump and long jump. This decrease in ROM explains the decline in functional performance also seen during these tests.

Key Words: Ankle sprain, functional performance tests, prophylactic ankle supports, ankle range of motion, motion analysis 
The lateral ankle sprain is one of the most common lower extremity injuries in sports. ${ }^{1-5}$ Repetitive jumping and landing in certain sports such as basketball and volleyball create an environment for increased risk of recurrent ankle injuries. ${ }^{6}$ The anterior talofibular ligament (ATFL) is the most commonly injured ankle ligament with $85 \%$ of ankle injuries involving the ATFL. Of greater concern, once a person sustains an ankle sprain, they are more likely to suffer a recurrent sprain and have residual instability. ${ }^{6}$ Recurrent sprains can compromise the integrity of the ankle joint, causing the athlete to have feelings of the ankle "giving way."

One common strategy used to reduce the frequency of ankle sprains is to apply prophylactic ankle support to the joint. Authors of previous research have also identified that wearing some type of external prophylactic ankle support reduces the risk of recurrent ankle sprains and provides extra support to the joint. ${ }^{2,4,7}$ The purpose of using an external prophylactic brace is to provide support to the joint through restricting inversion and eversion movements. This additional support to the ankle joint can help limit the amount of stress applied to the ligaments and potentially reduce the risk of inversion or eversion injury to the ankle., ${ }^{4,8}$

There are a variety of external prophylactic ankle braces that can be used, but the most commonly used braces are the lace-up and semi-rigid braces. ${ }^{3,6}$ Some of the major advantages of ankle braces are that they are reusable, adjustable, and washable. However, some athletes believe that wearing an ankle support may negatively impact their athletic performance..$^{7,9,10}$

Ankle braces are constructed using different materials and design philosophies; the support levels will vary between designs that may influence range of motion restriction and ultimately athletic performance. ${ }^{11,12}$ More specifically, some braces cover more of the forefoot while others focus the support solely around the talocrural joint. When the forefoot is covered by 
the ankle brace, more plantarflexion range of motion may be restricted resulting in a decrease in performance particularly during the vertical jump. ${ }^{4}$

Laboratory based studies, which evaluated how braces influence functional performances have led to mixed results. ${ }^{4,7,10,11,13}$ The majority of the studies ${ }^{7-19}$ have evaluated the influence of ankle braces during a variety of functional tests such as the vertical jump, agility drills and speed tests. These studies usually evaluate the effect of the brace only on performance measures such as the height, distance or speed for these tests, respectively. Therefore, when authors conclude that ankle bracing restricts overall performance, they can only hypothesize as to why the diminished performance occurs because motion in the primary plane dictating performance was not measured. Many suggest the reduction in performance is due to restriction in the range of motion of the joint, but few studies ${ }^{1,20,21}$ have actually captured kinematic data during the testing. The purpose of this study is twofold. First, to determine if two types of ankle braces have an effect on agility, speed, and/or vertical jump performance compared with no-brace. Second, to determine if ankle joint angles in the primary plane of motion differ between the braced and nobraced conditions at certain points in the functional performance tests.

\section{Methods}

\section{Participants}

Twenty participants (11 females, 9 males, $20.6 \pm 1.62$ yrs., $1.77 \pm 0.09 \mathrm{~m}, 74.75 \pm 14.44$ $\mathrm{kg}$ ) who were involved in at least one hour of physical activity (e.g. sport activity, cardiovascular exercise, weight training) five days a week participated in the study. Participants were included if they had not sustained a lower extremity injury within the past six weeks; however, all participants were injury free for the previous 12 months. All participants were either former or current high school or Division 1 college athletes. Participants participated in the following 
sports: tennis ( $\mathrm{n}=7 ; 1$ male, 6 female), volleyball $(\mathrm{n}=3 ; 0$ male, 3 female), basketball $(\mathrm{n}=8 ; 7$ male, 1 female) and soccer ( $\mathrm{n}=2 ; 1$ male, 1 female). All participants read and signed an informed consent form prior to participating in the study that was approved by the university's Institutional Review Board on use of human participants. All rights of the participants were protected.

\section{Procedures}

Experimental Design. All participants completed one day of testing. The participants performed four different functional performance tests: standing vertical jump, T-drill (or Tagility test), 40-yard sprint, and the standing long jump. Although participants had previous experience performing the functional performance tests prior to testing, all participants practiced each functional performance test until they were comfortable with the procedures. Further, feedback was provided regarding how to complete each test properly. The functional performance tests were performed in three conditions: a traditional lace-up brace (brace 1), a modified lace-up brace (brace 2), and no-brace. Both ankles of the participant were braced during the testing but kinematic data was collected only on the participant's dominant leg. Leg dominance was determined by asking with which leg they prefer to kick a ball. All participants wore a standardized shoe (Adidas barricade team 3) while performing the functional performance tests. To ensure accurate measurement of the inversion joint angle rather than the motion of the shoe during cutting portion of the T-drill, a rectangular window was cut out from the posterior heel counter within the shoe upper in order to place the reflective markers directly over the participant's calcaneus. The window did not affect the integrity of the heel counter or midsole thus any effect of the window on the shoe-brace-foot interaction was minimal. Markers were placed away from the edges of the window so the shoe would not interfere with the marker 
position. Following each functional performance test, participants completed a questionnaire about how they perceived the ankle brace affected their performance as well as questions related to their comfort while wearing the brace. During this time, participants sat to rest while the ankle brace was changed. The next set of functional performance tests did not start until the participants indicated they were ready to continue. The order of functional tests and brace conditions were counterbalanced across all participants. The participant completed three trials of each functional performance test in brace each condition. Participants did not perform the next trial within or between conditions until they felt rested and ready to continue. Performance outcomes were the maximum height (vertical jump), maximum distance (standing long jump), and time to completion (T-drill, 40 yard sprint) and were used for statistical analysis to measure. Prophylactic Conditions. Brace 1 (ASO EVO, Medical Specialties Inc. Charlotte, NC) and brace 2 (ASO Quatro, Medical Specialties Inc. Charlotte, NC) are lace-up ankle braces with two internal straps, medial and lateral, that are pulled to the outside of the brace. The dynamic cuff encircles the lower leg. The ankle brace can be retightened using the stabilizing straps without having to remove the shoe. By removing the dynamic cuff to expose the stabilizing straps the athlete can pull the loops to tighten the stabilizing straps. Brace 1 is a traditional lace-up ankle brace and brace 2 is a modified lace-up brace with a lower profile over the inferior tibiofibular joint. For the no-brace condition, participants completed the functional performance tests wearing only the standardized shoe, which was also used in the brace conditions.

Functional Performance Tests. Vertical jump was measured using a Vertec. Participants performed three trials and the maximum jump height was used for statistical analysis. For the standing long jump, participants started from a stationary stance with both feet behind a starting line. Participant were instructed to jump forward as far as possible, using only the swing of the 
arms as a counterforce. The long jump distance was measured from the starting line to the where the heel landed. Three successful long jump trials were retained for analysis. All participants adopted a similar overall movement strategy by performing a counter-movement for the vertical and long jumps. The T-drill was used to assess the agility and speed of the participant, the fastest time was used for statistical analysis. T-drill and 40-yard sprint time were measured with two photocell timing gates. The height of timing gates were adjusted to match the height of the participant's pelvis. The fastest of the three trials was used for statistical analysis. Apart from these instructions and those described below for the motion capture, participants performed all of the functional performance tests correctly, as they saw fit and performed them the same way between brace 1, brace 2, and the no-brace conditions.

Motion Capture. An infrared high speed motion capture camera (Innovision Systems, Inc., Columbiaville, MI, USA) collecting at $120 \mathrm{~Hz}$ was used to record two-dimensional ankle angles the primary plane of motion dictating performance during the vertical jump, standing long jump and T-drill. Motion capture was not recorded during the 40-yard sprint. The camera was placed $3.5 \mathrm{~m}$ perpendicular from the participant, at a height of $0.5 \mathrm{~m}$ from the floor and remained in the same place for the entire testing process. The camera was aligned in parallel to the plane of motion of interest for each functional performance test and trials were repeated if the participant performed a test out of alignment with the camera. Although restricting participant motion to be in parallel with the camera may have effected optimal performance of each test, it improved the accuracy of the captured data and this constraint effected all participants and conditions systematically. MaxTRAQ software (Innovision Systems, Inc., Columbiaville, MI, USA) was used to digitize and track the reflective markers for the range of motion measurements during each functional performance test. 
For the vertical jump and standing long jump tests, the camera was aligned perpendicular to the sagittal plane of the participant to measure the primary motion dictating jump performance. Three spherical reflective markers were placed on the participant's dominant leg over the following landmarks: the lateral aspect of the tibial plateau, ${ }^{22}$ lateral malleolus, and the lateral aspect of the base of the $5^{\text {th }}$ metatarsal (Figure 1). Data were captured during the takeoff period of the vertical jump and standing long jump. Maximum sagittal plane range of motion was calculated in degrees as the non-normalized maximum minus the minimum dorsiflexion angle occurring between when the participants began the countermovement preparation of the jump to when the participants feet were no longer in contact with the ground (Figure 2 and Figure 3). ${ }^{23}$ This method allowed the full dorsiflexion to plantar flexion range of motion to be captured. The average of the three trials were used for statistical analysis. In order to standardize the jumping procedure, the participants were instructed to stand with their body perpendicular to the camera and stand behind a designated line. Before the participants performed the task, the participants' position was viewed on the computer and corrected by the tester if needed.

For the T-drill, the camera was placed perpendicular to the posterior frontal plane at the cone that the cutting maneuver was performed, cone "D" (Figure 4), given that inversion/eversion motion is the primary motion of the foot dictating performance during cutting. Rearfoot frontal plane motion was measured with four reflective markers on the following landmarks: two markers bisecting the calcaneus and two markers bisecting the lower third of the posterior leg over the distal soleus. Rearfoot inversion angle was measured as the angle between the line formed by the two markers placed over the calcaneus and the two markers placed on the posterior lower leg ${ }^{22}$ (Figure 1). During the cutting maneuver performed at cone "D" only, inversion range of motion in degrees was calculated by the non-normalized maximum minus the 
minimum joint angle during the period from the instant that the participant's foot planted at the cone to when the toe left the ground during the direction change (Figure 5). ${ }^{24}$ Inversion range of motion during the cutting maneuver of the T-drill was averaged across the three trials and used for statistical analysis.

\section{Statistical Analysis}

For the functional performance dependent variables, a Multivariate Analysis of Variance (MANOVA) with repeated measures (brace 1, brace 2, no-brace) was used. Follow-up univariate analysis of variance and pairwise comparisons were conducted on any significant findings. To evaluate the kinematic data, a single MANOVA with repeated measures (brace 1, brace 2, nobrace) was used to determine differences in maximal range of motion angles. Follow-up univariate analysis of variance and pairwise comparisons were conducted on any significant findings. For all analyses, a priori alpha level was set at $\mathrm{p}<0.05$.

\section{Results}

\section{Functional Performance Tests}

There was a significant difference in the power functional performance tests between the brace conditions $(\mathrm{p}=0.01)$. Follow-up univariate analysis identified a significant decrease functional performance between the braced conditions compared with no-brace for the vertical jump $(\mathrm{p}=0.01)$ and standing long jump $(\mathrm{p}=0.01)($ Figure $6 \mathrm{~A})$. Both brace conditions reduced vertical jump performance by a mean difference of $0.013 \mathrm{~m}$ compared with no-brace. Long jump performance was reduced by a mean difference of $0.093 \mathrm{~m}$ in brace 1 compared with no-brace and reduced by a mean difference of $0.089 \mathrm{~m}$ in brace 2 compared with no-brace (Figure $1 \mathrm{~A}$ ). 
No significant difference between conditions were identified for either the T-drill $(\mathrm{p}=0.57)$ or 40-yard sprint $(\mathrm{p}=0.35)$ (Figure 6B).

\section{Ankle Range of Motion}

Evaluation of the kinematic data supported our functional performance results by also establishing a significant difference between the conditions $(\mathrm{p}=0.01)$. Follow-up univariate analysis identified a significant difference in ankle range of motion between the conditions for both the vertical jump $(p=0.01)$ and standing long jump $(p=0.01)$, but not the T-drill $(p=$ 0.10). In the vertical jump, both brace conditions restricted range of motion in the sagittal plane by a mean difference of 7.8 degrees compared with the no-brace condition (Figure 7). Standing long jump performance was reduced by a mean difference of 6.7 degrees in brace 1 compared with no-brace and reduced by a mean difference of 7.5 degrees in brace 2 compared with nobrace (Figure 7). No significant difference was identified between conditions for the T-drill ( $p>$ $0.05)$.

\section{Discussion}

The primary findings of this study were that ankle braces have a negative effect on some power functional performance tests (i.e. vertical jump height and standing long jump distance), while performance on speed and agility measures (i.e. sprint and T-drill) were not affected. The kinematic findings assist in providing an explanation for the functional performance results. Sagittal plane motion during the power functional performance tests was restricted in the braced conditions, and therefore contributed to the decreased functional performance. However, the ankle braces did not restrict range of motion in the frontal plane, which may not prevent 
injurious motion. These findings suggest that ankle braces may limit performance without providing the intended clinical benefit.

The vertical jump is one of the most common tests used to measure power. However, the results of previous studies ${ }^{7-18}$ that investigated the influence of ankle braces on vertical jump performance are not conclusive. One topic that needs to be considered when evaluating the importance of vertical jump deficits is what magnitude of deficit should be considered clinically meaningful. In our study, we found that the $0.010-0.020 \mathrm{~m}$ decrease in vertical jump height was statistically significant which is also in agreement with previous studies. ${ }^{16,17}$ Conversely, a number of studies concluded that a vertical jump deficit of $0.010-0.030 \mathrm{~m}$ was not statistically significant. ${ }^{7,8,10-14}$

Additionally, our study revealed a $0.090 \mathrm{~m}$ difference in standing long jump performance between the brace and no-brace condition. In the study by Ozer, ${ }^{16}$ there was a mean difference in long jump performance of approximately $0.080 \mathrm{~m}$ between brace and no-brace conditions (nobrace $1.84 \pm 0.24 \mathrm{~m}$; brace $1.76 \pm 0.28 \mathrm{~m} ; \mathrm{p}=0.300$ ) yet this difference was not statistically significant. Despite these findings not being statistically significantly, the measured effect of the brace may be detrimental to the athletes' performance depending on whether the long jump is a sport specific task required for their respective sport. Based on this previous research, it appears that some ankle braces have an influence on vertical and long jump performance, but how much of a difference is clinically meaningful may depend on the sport and athlete. For example, a difference of 0.010-0.020 m may be meaningful for sports like volleyball, basketball and even a goalkeeper in soccer because any decrease in vertical jump height can be detrimental to their performance. 
There is limited research on the effect of ankle braces on standing long jump performance ${ }^{7,9,16}$ and the differences in the braces employed between studies may influence the results. For example, Pienkowski ${ }^{7}$ used more rigid stirrup (Aircast) brace, two lace up braces, and bare ankle and did not identify a difference in standing long jump distance performed. Burks $^{9}$ agreed with our findings and found a significant decrease in standing long jump distance performed when utilizing one lace up brace (Swede-O), but not the other lace up brace (Kallassy). Our study found that both the ASO EVO and ASO Quatro braces resulted in decreased standing long jump distance. Similar to the braces used in the present study, the Swede-O is a canvas lace-up with two vertical plastic supports on the lateral and medial aspect of the ankle. In contrast, the Kallassy is a soft neoprene brace with a figure-8 design that is adjusted with Velcro. The differences in the standing long jump distance between the Kallassy compared with the Swede-O and the braces used in the current study may be due to the lace-up design of these braces. The lace-up braces (e.g. ASO EVO, ASO Quatro, Swede-O and previous studies that apply to this statement) have been shown to restrict sagittal plane/plantarflexion motion because of the material crossing the talocrural axis ${ }^{17,25}$ although restricting sagittal plane motion is not the primary role of the brace. Restriction in the sagittal plane range of motion may interfere with the ability to generate high plantarflexion angular velocities and forces against the ground thus decrease the amount of power output during push-off for a jumping task. ${ }^{11,25}$ Additionally, the Kallassy brace may allow more plantarflexion motion as a result of the neoprene material being more flexible than the canvas of the Swede-O.

The current study is in agreement with a majority of previous literature ${ }^{7-9,11,13-15,17}$ that ankle braces do not have an influence on speed and agility performance, despite the different types of agility tests employed. This relative consensus is an important clinical finding as 
healthcare providers can confidently utilize prophylactic ankle supports without the concern that it will negatively affect speed and agility performance.

Ankle braces are used as a preventative measure against inversion/eversion ankle injuries by restricting excessive range of motion in the frontal plane and providing joint stability. ${ }^{2,4,5,9,10,12,15-17,26-28}$ It has been previously debated 1) whether braces restrict range of motion in the plane of interest and 2) whether that range of motion restriction negatively impacts performance. Numerous studies have been done to investigate the effect of ankle braces on performance, but few studies concurrently evaluated joint kinematics during the specific functional performance tests. Utilizing kinematic analysis can provide additional information and insight on the ankle joint to help explain any performance deficits or why there was no decrease in performance while wearing the brace.

Kinematic data for the vertical jump and standing long jump were captured until the point of take-off. During both the standing long jump and vertical jump, the difference in dorsiflexion to plantar flexion range of motion between the brace and no-brace conditions was approximately $7^{\circ}$. Similarly, previous work also found plantarflexion deficits $\left(+3.0^{\circ}\right.$ to $\left.+21.3^{\circ}\right)$ and dorsiflexion deficits $\left(-9.8^{\circ}\right.$ to $\left.+10.5^{\circ}\right)$ with the ankle braces. ${ }^{1,5,17,20,21}$ In the current study, we suggest that this decrease in range of motion negatively impacted vertical jump height. Decreased sagittal plane motion may limit the athlete's ability to produce the necessary power to push off for jumping tasks. ${ }^{26,29}$ According to Cordova ${ }^{26}$, the lace-up brace covers the talocrural joint axis anteriorly and posteriorly which can interfere with plantarflexion motion. While trying to limit excess inversion and eversion, it appears that these lace-up ankle braces are restricting more plantarflexion and dorsiflexion which may cause a decrease in power performance. We hypothesized that brace 1 (ASO EVO) would negatively impact jump distance based on the 
amount of forefoot coverage. Brace 2 (ASO Quatro) has less forefoot coverage, which in theory would allow less dorsiflexion and plantarflexion restriction compared with brace 1. Despite the design difference, both braces yielded similar kinematic and performance results. Aside from the difference in forefoot coverage, both braces have the same stirrup and stabilizing system to provide support to the subtalar and talocrural joints. With these straps working to keep excessive motion from occurring in the subtalar and talocrural joints it may be the reason why both braces had similar results.

Despite the purpose of braces to restrict inversion/eversion ankle motion, our findings for the frontal plane kinematics during the T-drill were not significantly different between the three conditions. The mean difference in inversion range of motion between the braced and no-brace condition was only $3^{\circ}(p=0.10)$. Similarly, Zhang ${ }^{30}$ did not find a significant difference between the ASO brace $\left(27.3 \pm 5.8^{\circ}\right)$ and no-brace condition $\left(26.7 \pm 6.0^{\circ}\right)$ during the lateral cutting task. Conversely, Parsely ${ }^{17}$ used the same traditional lace-up brace as our study and found inversion range of motion significantly decreased by $5.4^{\circ}$ compared with the no-brace condition during nonweight-bearing active range of motion testing. The minimal difference in frontal plane restriction seen in our study between the braced and no-brace conditions might be why the performance on the agility functional performance tests was similar.

At the end of each braced condition, participants completed a brief questionnaire about their performance wearing the ankle braces as well as comfort of the braces. While wearing brace $1,30 \%$ of the participants felt the brace restricted their vertical jump and 40 -yard sprint performance compared with the no-brace condition. Thirty-five percent felt brace 1 restricted ability to perform the T-drill. The most restriction felt was during the standing long jump; $40 \%$ of the participants felt brace 1 affected their ability to perform the jumping task. For brace 2, 
$20 \%$ of the participants stated that they felt brace 2 restricted their performance during the vertical jump and 40-yard sprint compared with the no-brace condition. Forty-five percent of the participants felt their ankle was restricted when wearing brace 2 while performing the T-drill compared with the no-brace condition. Only $25 \%$ found brace 2 to restrict their standing long jump performance.

Athletes that perform jumping tasks may be resistant to the idea of wearing ankle braces if it will decrease their jumping height or distance. The results of the current study show that vertical jump was decreased by $0.01-0.02 \mathrm{~m}$ and the standing long jump was decreased by 0.09 m. Even though there are no performance differences between the two braces, the modified laceup brace may appeal more to these athletes because qualitatively, participants reported that brace 2 did not restrict or inhibit their performance compared with brace 1 . The perception of whether a brace will or will not affect performance may influence the athlete's decision to wear the ankle brace. An athlete may still be resistant to wearing ankle braces if they feel that the brace is too bulky or heavy. Given that the two braces used in the present study resulted in very similar findings, the athletic trainer should consider sport and athlete preference when determining the best brace option.

\section{Limitations}

The use of a two-dimensional camera system limits measurement to only one plane and requires that the motion of interest is directly aligned perpendicular to the plane of the camera. Two-dimensional motion capture increases the likelihood of perspective error compared with three-dimensional motion capture, which may affect the magnitude of the angle measured. The T-drill will be affected more by perspective error than the vertical jump or long jump given that 
the T-drill is a more dynamic, multi-planar movement. To reduce the likelihood of perspective error, we aligned the motion of the performance tests as much as possible with the camera's field of view and repeated trials if the participant performed a test out of alignment with the camera. The use of three-dimensional motion capture would eliminate the effect of perspective error; however, three-dimensional systems are more expensive, not as portable, and more difficult to implement in the field.

There is some debate on whether shoes should be worn with the ankle braces when testing ankle kinematics because movement of the shoe may not reflect movement of the foot and shoes may alter the effects of the brace. Removing the shoe will allow for direct measurement of foot movement because the reflective markers would be attached directly to the skin over the foot rather than the shoe. ${ }^{8,21}$ Alternatively, we cut the shoe upper so the reflective markers could be secured to the skin over the calcaneus. This method allows measurement of foot motion while maintaining the construct validity of wearing shoes during athletic performance. Similarly, wearing shoes with ankle braces is more clinically relevant although testing ankle braces barefoot would eliminate any effects the shoe has on either the ankle or brace.

\section{Conclusion}

The primary findings of this study revealed ankle braces have a negative effect in both vertical jump height and standing long jump distance while speed and agility performance was not affected. The kinematic findings provide a potential rational to explain the performance outcomes. During the power functional performance tests, sagittal plane motion was restricted in the braced conditions and could have therefore contributed to the decreased jump performance. 
Interestingly, the braces limited range of motion in the sagittal plane but did not restrict range of motion in the plane that ankle injury occurs (i.e. frontal plane). 


\section{References}

1. Chen Q, Wortley M, Bhaskaran D, Milner CE, Zhang S. Is the inverted surface landing more suitable in evaluating ankle braces and ankle inversion perturbation? Clin J Sport Med. 2012;22(3):214-220.

2. Forbes H, Thrussell S, Haycock N, Lohkamp M, White M. The effect of prophylactic ankle support during simulated soccer activity. J Sport Rehab. 2013;22(3):170-176.

3. Verhagen EALM, van der Beek AJ, Willem van Mechelen MD. The effect of tape, braces and shoes on ankle range of motion. Sports Med. 2001;31(9):667-677.

4. Bot SDM, van Mechelen W. The effect of ankle bracing on athletic performance. Sports Med. 1999;27(3):171-178.

5. Alfuth M, Klein D, Koch R, Rosenbaum D. Biomechanical comparison of 3 ankle braces with and without free rotation in the sagittal plane. J Ath Train. 2014;49(5):608-616.

6. Swenson DM, Collins CL, Fields SK, Comstock RD. Epidemiology of us high school sports-related ligamentous ankle injuries. Clin J Sport Med. 2013;23(3):190-196.

7. Pienkowski D, McMorrow M, Shapiro R, Caborn DNM, Stayton J. The effect of ankle stabilizers on athletic performance - A randomized prospective study. Am J Sports Med. $1995 ; 23(6): 757-762$.

8. Yaggie JA, Kinzey SJ. A comparative analysis of selected ankle orthoses during functional tasks. J Sport Rehab. 2001;10(3):174-183.

9. Burks RT, Bean BG, Marcus R, Barker HB. Analysis of athletic performance with prophylactic ankle devices. Amer J sports Med. 1991;19(2):104-106. 
10. Ambegaonkar JP, Redmond CJ, Winter C, et al. Ankle stabilizers affect agility but not vertical jump or dynamic balance performance. Foot Ankle Specialist. 2011;4(6):354360.

11. Mackean LC, Bell G, Burnham RS. Prophylactic ankle bracing vs taping- Effects on Fucntional Performance in Female Basketball Players. J Orthop Sports Phys Ther. 1995;22(2):77-81.

12. Rosenbaum D, Kamps N, Bosch K, Thorwesten L, Völker K, Eils E. The influence of external ankle braces on subjective and objective parameters of performance in a sportsrelated agility course. Knee Surg, Sports Traumatol, Arthrosc. 2005;13(5):419-425.

13. Macpherson K, Sitler,Michael Kimura,Iris, Horodyski,MaryBeth. Effects of a semirigid and softshell prophylactic ankle stabilizer on selected performance tests among high school football players. J Orthop Sports Phys Ther. 1995;21(3):147-152.

14. Bocchinfuso C, Sitler M, Kimura I. Effects of two semirigid prophylactic ankle stabilizers on speed, agility, and vertical jump. J Sport Rehab. 1994;3(2):125-134.

15. Hals TMV, Sitler MR, Mattacola CG. Effect of a semi-rigid ankle stabilizer on performance in persons with functional ankle instability. J Orthop Sports Phys Ther. 2000;30(9):552-556.

16. Ozer D, Senbursa G, Baltaci G, Hayran M. The effect on neuromuscular stability, performance, multi-joint coordination and proprioception of barefoot, taping or preventative bracing. Foot. 2009;19(4):205-210.

17. Parsley A, Chinn L, Lee SY, Ingersoll C, Hertel J. Effect of 3 different ankle braces on functional performance and ankle range of motion. Athl Train Sports Health Care. $2013 ; 5(2): 69-75$ 
18. Saldanha dos Anjos MTS, Brasil LJ, Barros BD, Bastos NCD, Moraes GFD. Effect of the use of the active ankle system stabilizer in the vertical jump height in volleyball players. Rev Bras Med Esporte. 2009;15(5):347-350.

19. Verbrugge JD. The effects of semirigid air-stirrup bracing vs. adhesive ankle taping on motor performance. J Orthop Sports Phys Ther. 1996;23(5):320-325.

20. DiStefano LJ, Padua DA, Brown CN, Guskiewicz KM. Lower extremity kinematics and ground reaction forces after prophylactic lace-up ankle bracing. J Ath Train. 2008;43(3):234-241.

21. Gudibanda A, Wang Y. Effect of the ankle stabilizing orthosis on foot and ankle kinematics during cutting maneuvers. Resear Sports Med. 2005;13(2):111-126.

22. Cornwall M, McPoil T. Comparison of 2-dimensional and 3-dimensional rearfoot motion during walking. Clin Biomech. 1995;10(1):36-40.

23. Wakai M, Linthorne NP. Optimum take-off angle in the standing long jump. Human Movement Sci. 2005;24(1):81-96.

24. Wheeler KW, Sayers MG. Modification of agility running technique in reaction to a defender in rugby union. J Sports Sci Med. 2010;9(3):445.

25. Cordova ML, Scott BD, Ingersoll CD, LeBlanc MJ. Effects of ankle support on lowerextremity functional performance: a meta-analysis. Med Sci Sports Exer. 2005;37(4):635641.

26. Cordova ML, Ingersoll CD, LeBlanc MJ. Influence of ankle support on joint range of motion before and after exercise: A meta-analysis. J Orthop Sports Phys Ther. 2000;30(4):170-177. 
27. Nisikawa T, Kurosaka M, Mizuno K, Grabiner M. Protection and performance effects of ankle bracing. Inter Ortho. 2000;24(5):285-288.

28. Siegler S, Liu W, Sennett B, Nobilini RJ, Dunbar D. The three-dimensional passive support characteristics of ankle braces. J Orthop Sports Phys Ther. 1997;26(6):299-309.

29. Quackenbush KE, Barker PRJ, Stone Fury SM, Behm DG. The effects of two adhesive ankle-taping methods on strength, power, and range of motion in female athletes. $N$ Ameri J sports PT. 2008;3(1):25-32.

30. Zhang S, Wortley M, Chen Q, Freedman J. Efficacy of an ankle brace with a subtalar locking system in inversion control in dynamic movements. J Orthop Sports Phys Ther. 2009;39(12):875-883. 
Figure 1. Position of spherical reflective marker attachment for the (A) sagittal plane and (B) Frontal Plane. Markers were positioned over the following landmarks: the lateral aspect of the tibial plateau, lateral malleolus, and the lateral aspect of the base of the fifth metatarsal.

Figure 2. Kinematic analysis of plantarflexion and dorsiflexion of the ankle joint during the vertical jump using MaxTRAQ software. Range of motion was calculated in degrees from when the subjects began the countermovement preparation of the jump to when the subject's feet were no longer in contact with the ground.

Figure 3. Kinematic analysis of plantarflexion and dorsiflexion of the ankle joint during the standing long jump using MaxTRAQ software. Range of motion was calculated in degrees from when the subjects began the countermovement preparation of the jump to when the subject's feet were no longer in contact with the ground.

Figure 4. Schematic of the T-drill. The cones were set up in a "T" shape with three cones (B, C, D) in one horizontal line five yards apart from one another and a fourth cone (A), $9.14 \mathrm{~m}$ perpendicular to the middle cone. The subjects were instructed to touch the base of each cone before proceeding to the next one. The subjects started by sprinting from the starting line at cone A to the center cone $\mathrm{B}$, then laterally shuffled to the cone $\mathrm{C}$ on the left, then shuffled to the cone to the right $\mathrm{D}$, then laterally shuffled to the center cone $\mathrm{B}$, then backpedaled to the starting cone A. The motion capture camera was aligned perpendicular to either cone $\mathrm{C}$ or $\mathrm{D}$ in order to capture the plant and push-off of subject's dominate foot.

Figure 5. Kinematic analysis of inversion of the ankle joint during the T-drill using MaxTRAQ software. Inversion range of motion in degrees was calculated from the instant that the subject's foot planted at the cone to when the toe left the ground during the direction change.

Figure 6. Mean performance of the (A) the long jump and vertical jump functional performance tests and (B) the T-drill and 40 yard dash functional performance tests $(n=20)$. Error bars are +1 standard deviation. *Indicates a significant difference compared with the no-brace condition $(\mathrm{p}<$ $0.05)$.

Figure 7. Mean sagittal plane range of motion during the long jump and vertical jump functional performance tests and the mean frontal plane range of motion during the T-drill functional performance tests $(n=20)$. Error bars are +1 standard deviation. *Indicates a significant difference compared with the no-brace condition $(\mathrm{p}<0.05)$. 


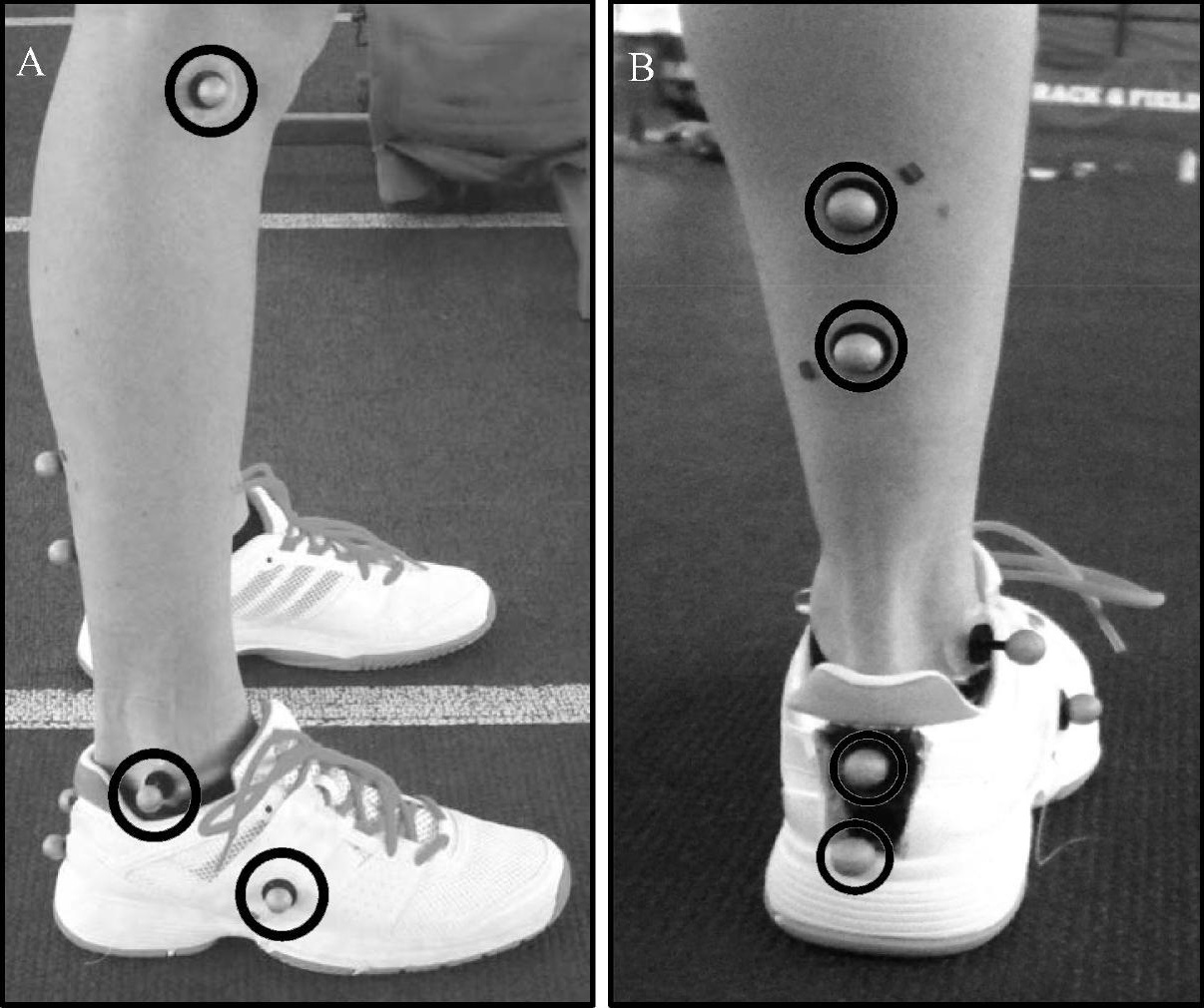




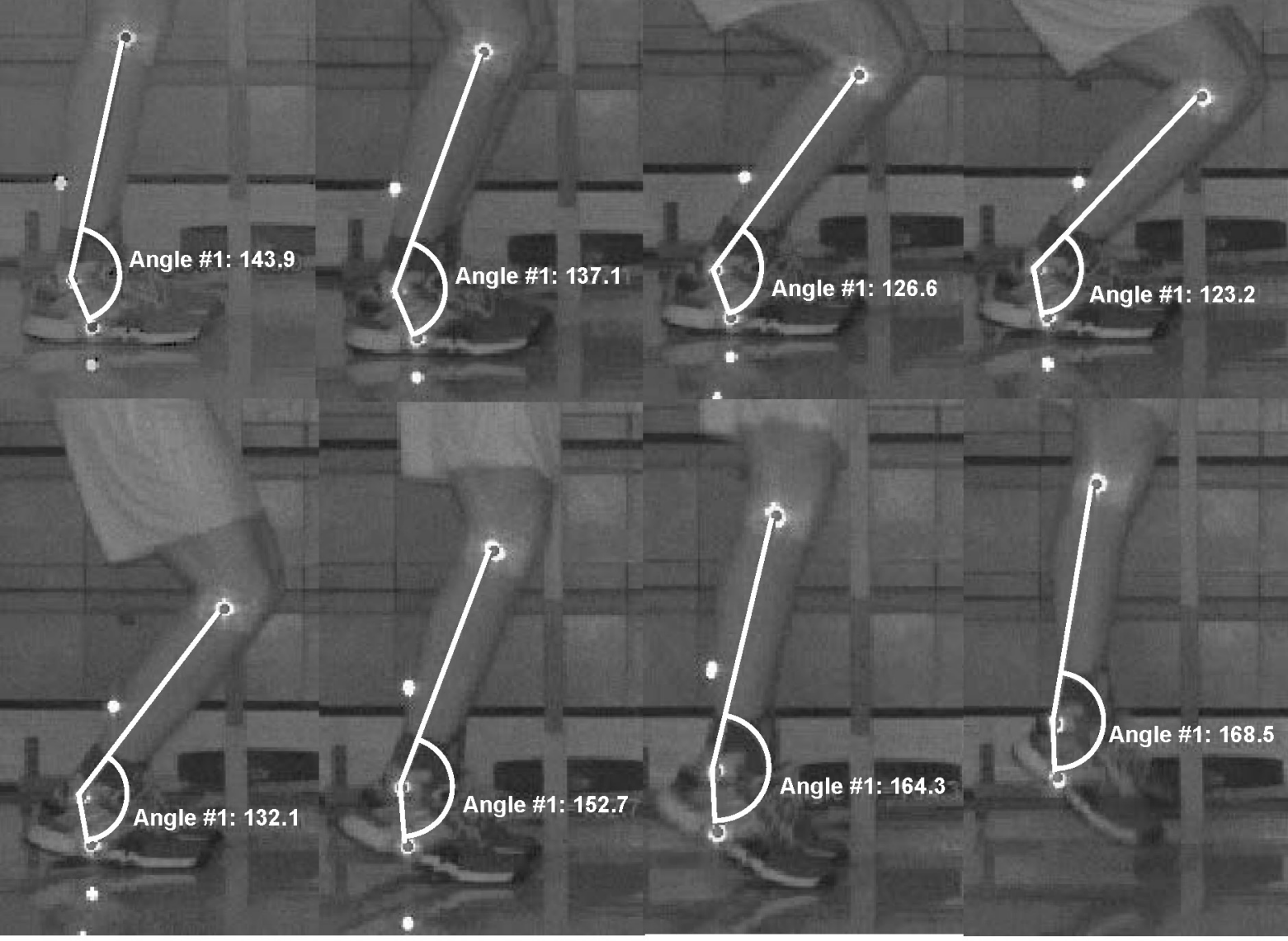



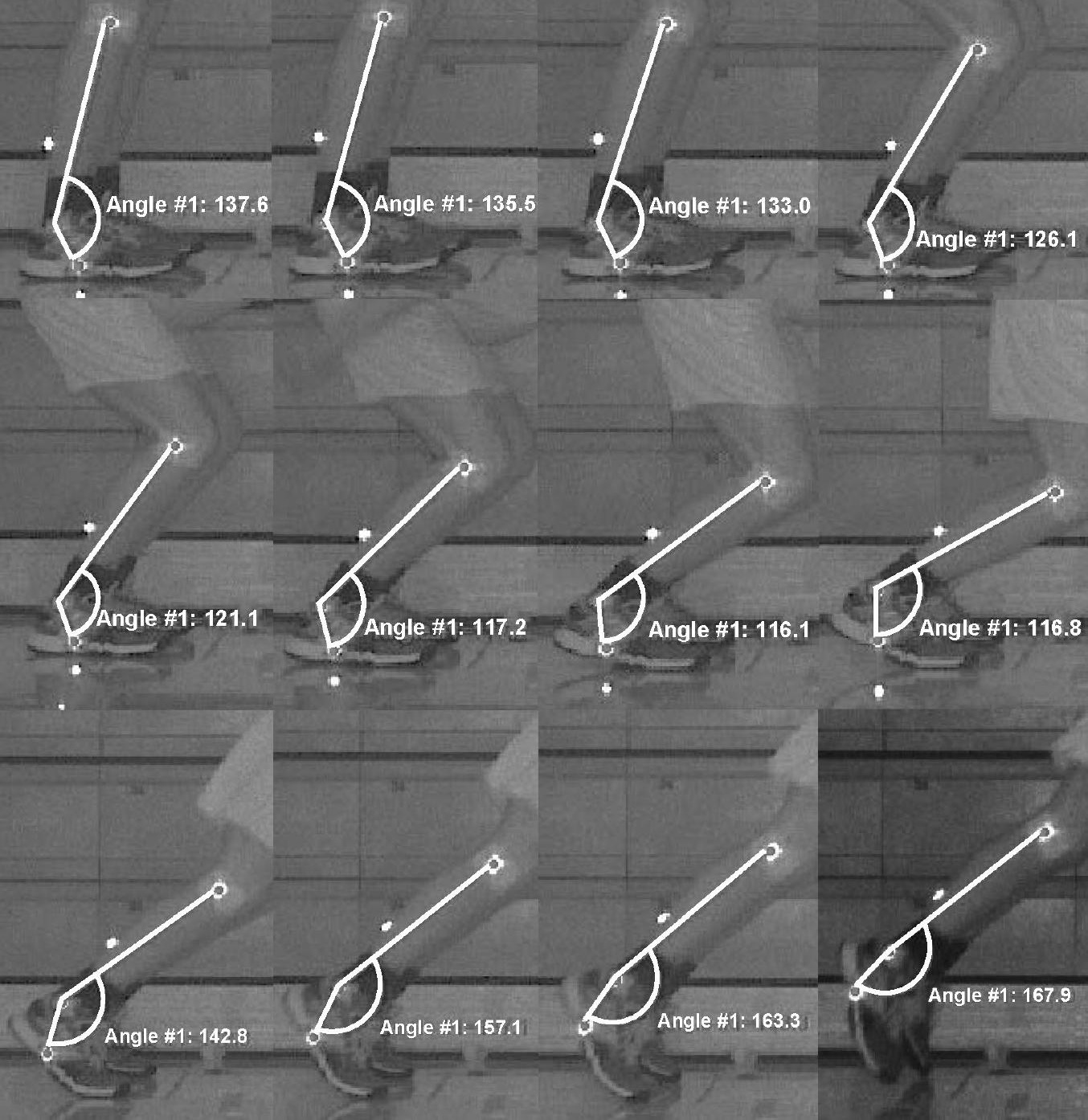


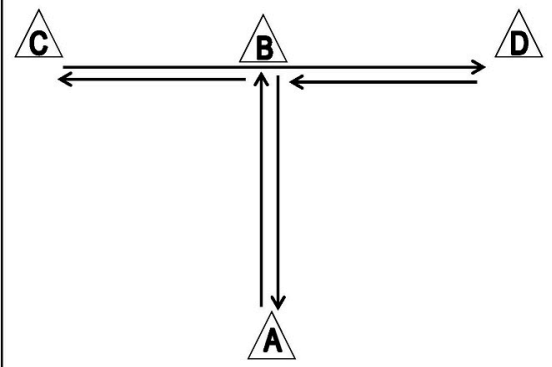



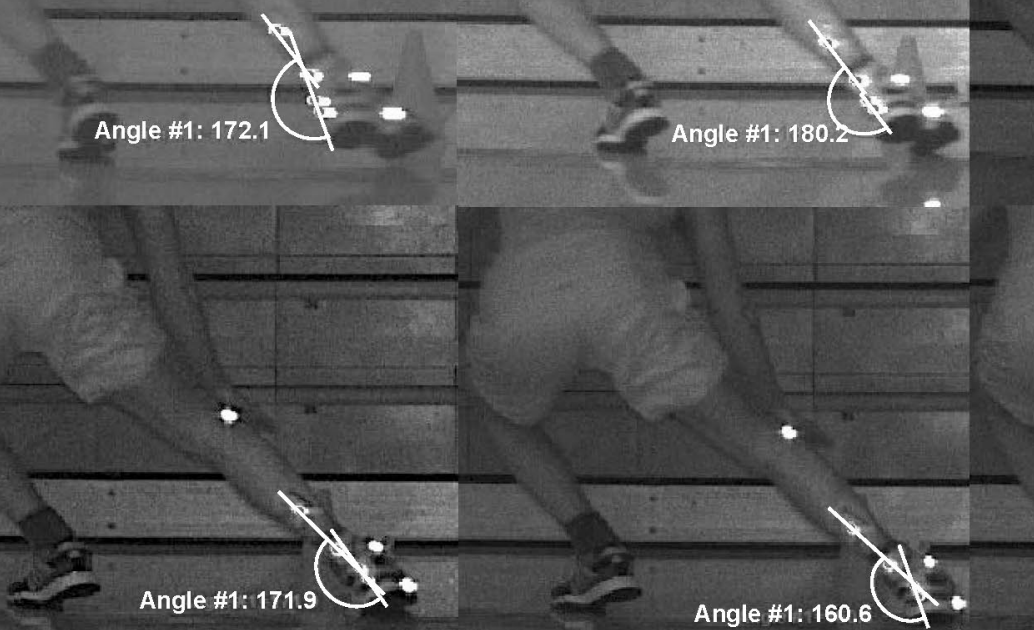

Angle \#1:171.9
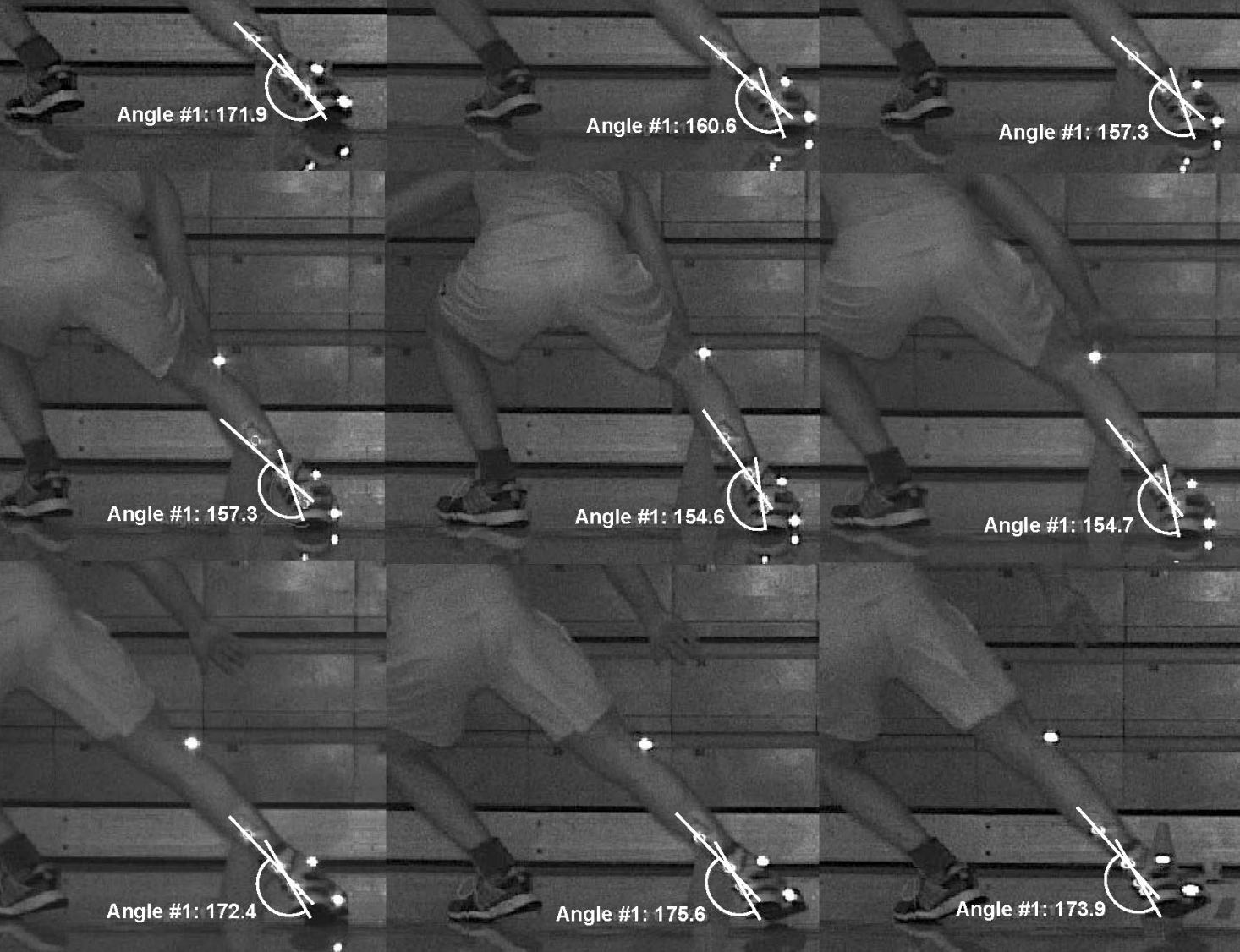
B)
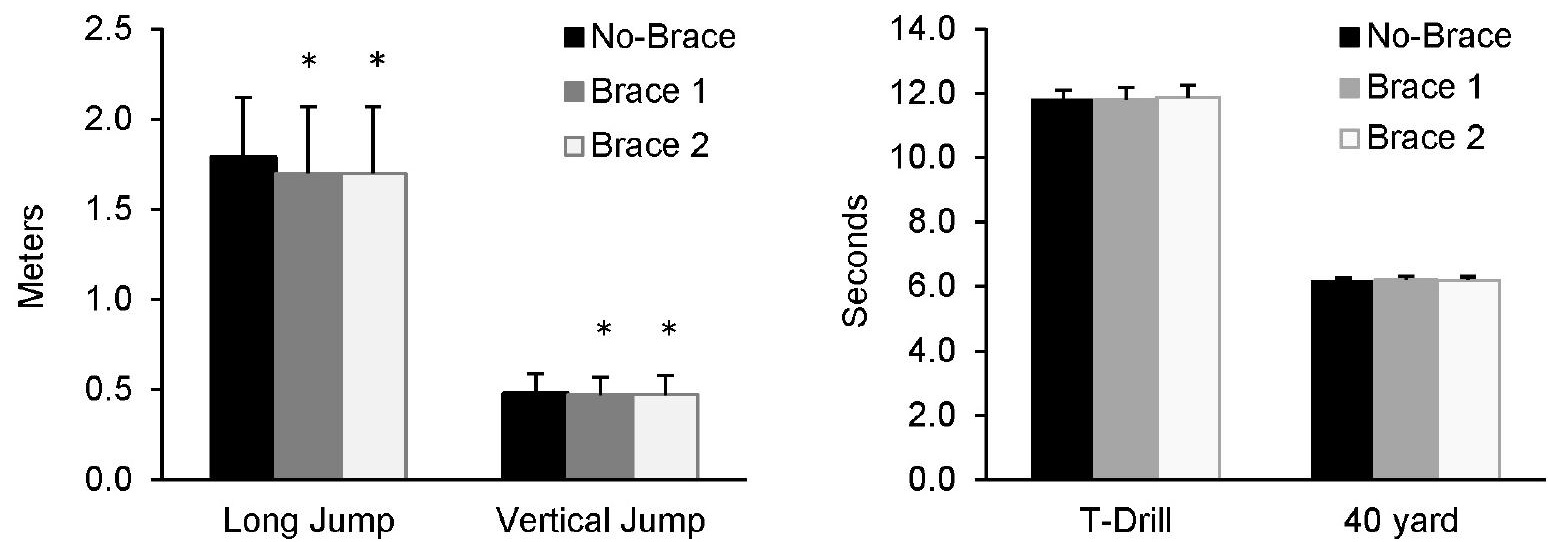


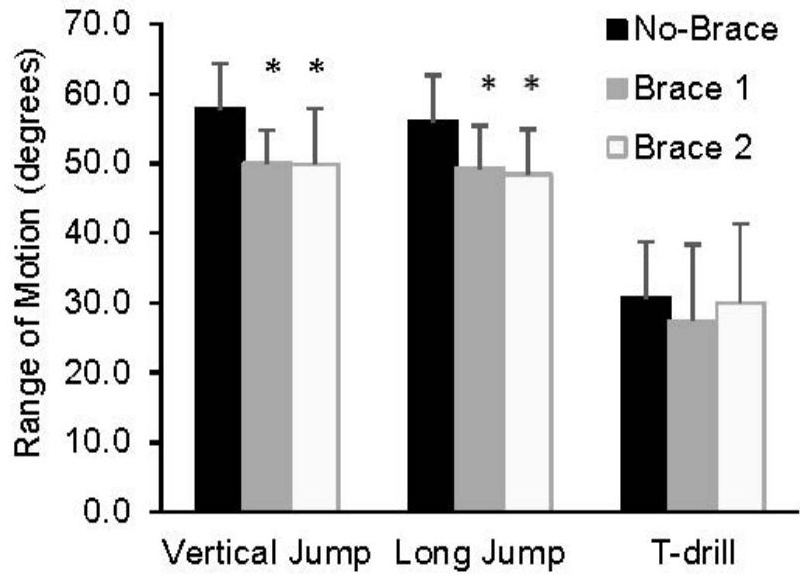

